Pure Appl. Chem., Vol. 81, No. 6, pp. 1065-1074, 2009.

doi:10.1351/PAC-CON-08-08-29

(c) 2009 IUPAC, Publication date (Web): 5 May 2009

\title{
Biosynthesis and biosynthetic engineering of calcium-dependent lipopeptide antibiotics*
}

\author{
Jason Micklefield \\ School of Chemistry and Manchester Interdisciplinary Biocentre, The University of \\ Manchester, 131 Princess Street, Manchester M1 7DN, UK
}

\begin{abstract}
Biosynthetic engineering involves the reprogramming of genes that are involved in the biosynthesis of natural products to generate new "non-natural" products, which might otherwise not exist in nature. Potentially this approach can be used to provide large numbers of secondary metabolite variants, with altered biological activities, many of which are too complex for effective total synthesis. Recently we have been investigating the biosynthesis of the calcium-dependent antibiotics (CDAs) which are members of the therapeutically relevant class of acidic lipopeptide antibiotics. CDAs are assembled by nonribosomal peptide synthetase (NRPS) enzymes. These large modular assembly-line enzymes process intermediates that are covalently tethered to peptidyl carrier protein (PCP) domain bonds, which makes them particularly amenable to reprogramming. The CDA producer, Streptomyces coelicolor, is also a genetically tractable model organism which makes CDAs an ideal template for biosynthetic engineering. To this end, we have elucidated many of the key steps in CDA biosynthesis and utilized this information to develop methods that have enabled the engineered biosynthesis of a wide range of CDA-type lipopeptides.
\end{abstract}

Keywords: biosynthesis; lipopeptides; antibiotics; biosynthetic engineering; combinatorial biosynthesis.

\section{INTRODUCTION}

The calcium-dependent antibiotics (CDAs) are nonribosomally biosynthesized, cyclic lipopeptides produced by Streptomyces coelicolor (Fig. 1). CDAs belong to the acidic lipopeptide group of antimicrobial agents which include friulimicin, amphomycin, and daptomycins. These lipopeptides all possess $N$-terminal fatty acid acyl chains and decapeptide lactone or lactam cores, with a number of acidic amino acid residues that coordinate calcium ions which are essential for antimicrobial activity. In 2003, daptomycin was approved for clinical use in the treatment of skin and soft tissue infections, caused by Gram-positive pathogens including methicillin- and vancomycin-resistant Staphylococcus aureus. As a result, there has been considerable interest in understanding how the acidic lipopeptides are biosynthesized. In particular, it is envisaged that this knowledge could enable the engineered combinatorial biosynthesis [1] of new lipopeptides, with improved activity, to combat the emergence of pathogens with resistance to current antibiotic treatments.

\footnotetext{
*Paper based on a presentation at the $26^{\text {th }}$ International Symposium on Chemistry of Natural Products (ISCNP-26) and $6^{\text {th }}$ International Conference on Biodiversity (ICOB-6), 13-18 July 2008, Charlottetown, Prince Edward Island, Canada. Other presentations are published in this issue, pp. 1001-1129.
} 


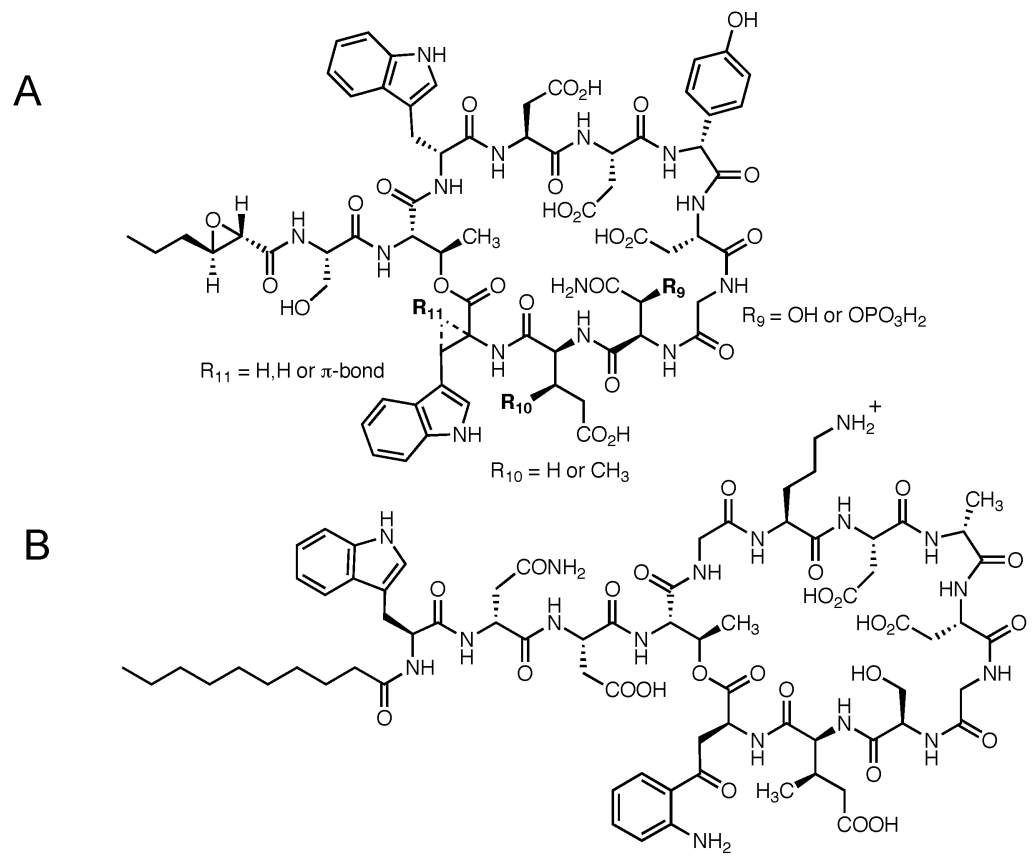

Fig. 1 (A) Structures of wild-type CDA variants from S. coelicolor. (B) Structure of daptomycin produced by $S$. roseosporus.

Previously, we solved the solution structures of daptomycin using NMR and showed that the lipopeptide coordinates $\mathrm{Ca}^{2+}$ with 1:1 stoichiometry resulting in aggregation [2]. These findings were consistent with the proposed mechanism of action, which suggested that upon coordination to $\mathrm{Ca}^{2+}$ daptomycin aggregates and penetrates into the cytoplasmic membrane of Gram-positive bacteria. The subsequent disruption of the membrane results in the loss of potassium ions, depolarization of membrane potential, and ultimately cell death [3] (Fig. 2A). More recent studies have revealed that CDAs, in addition to possessing a similar primary structure, adopt a 3D structure that is also similar to daptomycin consistent with a common mode of action. In light of this knowledge derived from studies on CDA biosynthesis, engineering and structure-activity relationship can be applied to the more therapeutically relevant daptomycins.

\section{DISCUSSION}

\section{Origins of the nonproteingenic amino acid precursors}

In order to develop the biosynthetic engineering methodology required to produce "non-natural" lipopeptides, it was first necessary to elucidate the key steps in the biosynthetic pathways leading to the nonproteinogenic amino acids and other precursors. To this end, we were greatly aided, at the outset, by the completion of the $S$. coelicolor genome sequencing project. This revealed the $c d a$ biosynthetic cluster which encompasses $82 \mathrm{~kb}$ of the chromosome [4]. At the center of the cluster are 3 large open reading frames (ORFs) encoding the nonribosomal peptide synthetase (NRPS) assembly-line enzymes (CdaPS1, 2, and 3). On either side of these ORFs are genes predicted to be involved in resistance and regulation, as well as genes encoding enzymes involved in the biosynthesis of the amino acid and fatty acid precursors (Fig. 2B) [4]. 

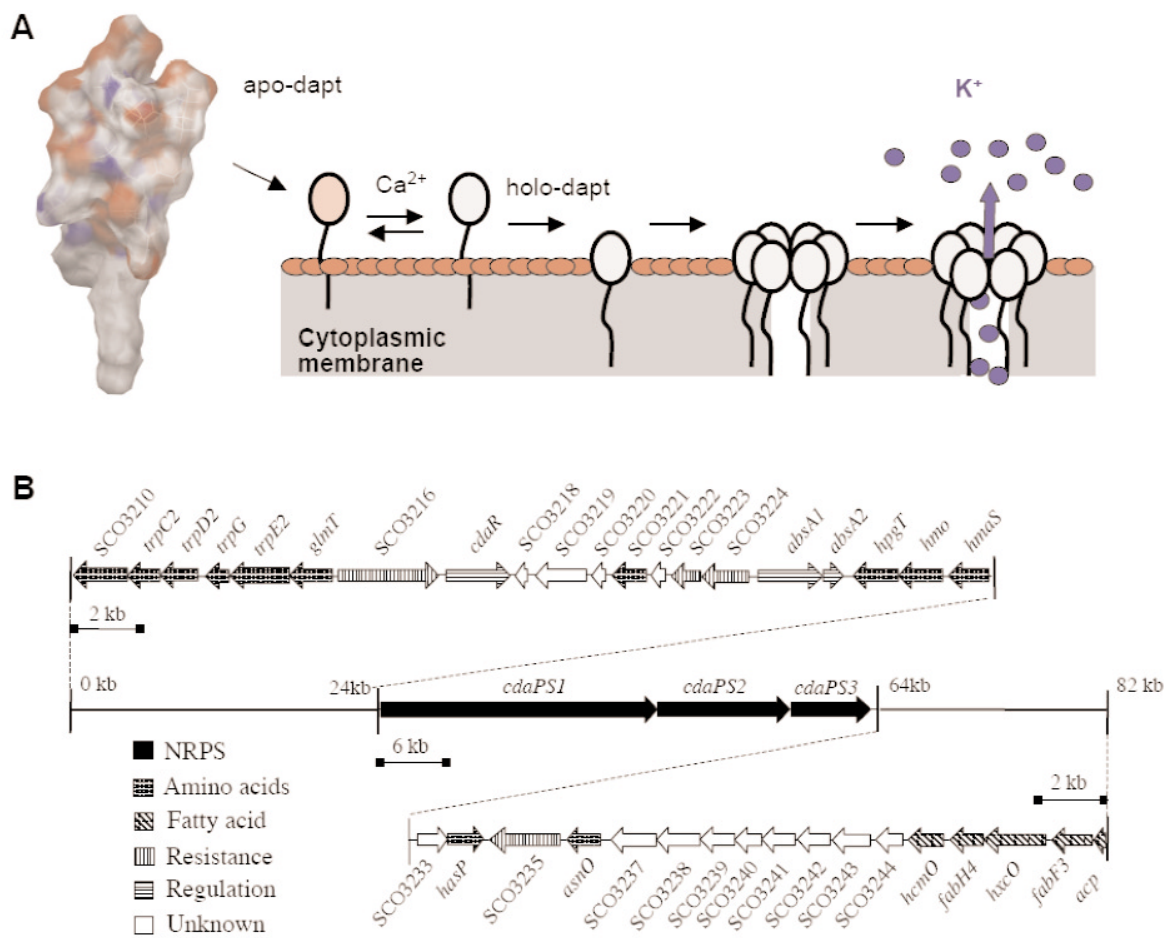

Fig. 2 (A) Structure [2] and proposed mechanism of action for daptomycin [3]. (B) The cda biosynthetic gene cluster [4].

Our initial focus was the biosynthesis of the hydroxyphenylglycine (HPG) residue at position 6 in CDA. Studies from other laboratories [5] had suggested a pathway to HPG (Fig. 3A). In order to test if this pathway was operative in S. coelicolor, the gene $h m a S$ was deleted from the cda biosynthetic gene cluster. HmaS was predicted to encode 4-hydroxymandelate synthase (HmaS) the first dedicated enzyme in HPG biosynthesis. As anticipated, CDA production was abolished in the deletion mutant $(\Delta h m a S)$, and was then re-established on feeding the putative intermediates $(\mathbf{2}-\mathbf{4}, \mathrm{X}=\mathrm{OH})$. This confirmed that the predicted pathway for HPG biosynthesis is also operative in S. coelicolor. Moreover, by feeding analogs (2-4, $\mathrm{X}=\mathrm{H}$ or $\mathrm{F})$ to $\Delta h m a S$ it was possible to affect the mutasynthesis of new CDAs with modified arylglycine residues (Fig. 7: CDA2d, 2fa, 2fb) [4].

Following this, we set about elucidating the biosynthesis of 3-methylglutamic (3-MeGlu) residue, which is found at the same relative positions in both CDAs and daptomycin and is known to be important for antimicrobial activity. Firstly, we completed the stereocontrolled synthesis of $(2 S, 3 S)$ - and $(2 S, 3 R)-3-$ MeGlu and showed by high-performance liquid chromatography (HPLC) that 3-MeGlu samples derived from hydrolysis of daptomycin and CDAs both possess the $2 S, 3 R$ configuration [6]. A methyltransferase-encoding gene $(g \operatorname{lm} T)$, identified within the $c d a$ cluster [4], was then deleted. The resulting $\Delta g \operatorname{lm} T$ mutant was shown to produce Glu containing CDA3a, with no 3-MeGlu containing CDAs evident. Complementation of the $\Delta g \operatorname{lm} T$ mutant with synthetic precursors 3-methyl-oxoglutarate 6 and $(2 S, 3 R)-3-M e G l u 7$ re-established production of 3-MeGlu containing CDA4a. This indicated that $\alpha$-ketoglutarate $(\alpha \mathrm{KG}, 5)$ is first methylated to give $(3 R)$-methyl-2-oxoglutarate $\mathbf{6}$ and then transaminated to (2S,3R)-3-MeGlu 7 prior to CDA lipopeptide-assembly (Fig. 3B). By screening a number of candidate enzymes, an aminotransferase (IlvE) encoded by a gene SCO5523 was shown to accept 3-methyl-2-oxoglutarate $\mathbf{6}$ as a substrate [7]. The two enzymes GlmT and IlvE were then shown to catalyze the in vitro transformation of $\alpha \mathrm{KG}$ to $(2 S, 3 R)-3$-MeGlu (Fig. 3B), which confirmed the earlier re- 


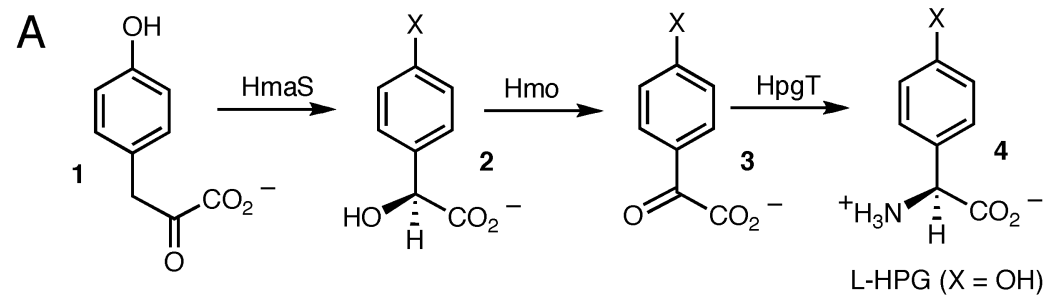

B

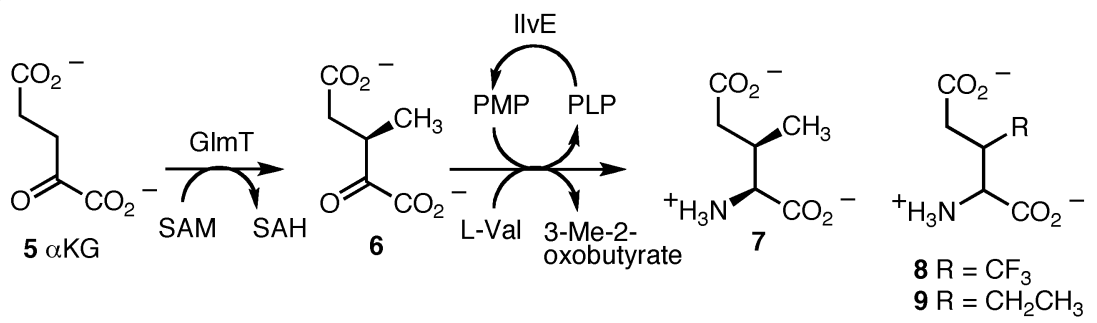

Fig. 3 (A) Biosynthesis of the HPG residue in CDAs, the natural intermediates all have para-hydroxyl groups (2-4 with $\mathrm{X}=\mathrm{OH})$. Analogs with other substituents at the para-position $(\mathbf{2}-\mathbf{4}$ with $\mathrm{X}=\mathrm{H}$, or $\mathrm{F})$, were used in mutasynthesis experiments. (B) Biosynthesis of the $(2 S, 3 R)-3$-methylglutamic acid residue in CDAs. IlvE is encoded by $S$. coelicolor gene SCO5523, which lies outside the $c d a$ biosynthetic gene cluster.

sults from in vivo experiments [6]. Presumably, the SAM-dependent GlmT-methylation of $\alpha \mathrm{KG}$ by the standard two-electron transfer mechanism is electronically more feasible, via a stabilized enol or enolate, than the direct methylation at the $\beta$-position of Glu. Based on our understanding of 3-MeGlu biosynthesis, a strategy was also developed for the mutasynthesis of lipopeptide variants possessing modified glutamate residues (Fig. 7: Et-CDA3b, $\mathrm{CF}_{3}-\mathrm{CDA} 3 \mathrm{a}$ and $3 \mathrm{~b}$ ) [8]. This involved feeding synthetic 3-trifluoromethyl and 3-ethyl glutamic acid derivatives $(\mathbf{8}, \mathbf{9})$ to the $\Delta g \operatorname{lm} T$ mutant strain.

At position 9 in CDA is found either $(2 R, 3 S)$-3-phosphohydroxyasparagine (3-OPAsn) or $(2 R, 3 S)$-3-hydroxyasparagine (3-OHAsn). Two genes were predicted to be involved in 3-OPAsn biosynthesis. The first, asn $O$, encodes a protein similar to the $\mathrm{Fe}(\mathrm{II}) / 2$-oxoglutarate-dependent oxygenase clavaminate synthase (CAS) which catalyzes $\beta$-hydroxylation of an Arg derivative during the biosynthesis of clavulanic acid. On this basis, we suggested that AsnO is an asparagine oxygenase that $\beta$-hydroxylates the Asn residue of CDA [9]. Downsteam of asnO is another gene that encodes a protein similar to a number of ATP-dependent phosphotransferases, which was suggested to phosphorylate the CDA 3-OHAsn residue. As predicted, deletion of the AsnO encoding gene results in a mutant strain $(\triangle a s n O)$, which produces new CDA variants possessing exclusively Asn residues at position 9 (CDA5b, 5a, 6a) [9]. Similarly, deletion of the putative phosphotransferase encoding gene resulted in a mutant $(\triangle h a s P$ ) which, when grown under conditions that favor phosphorylation of CDA, produces exclusively non-phosphorylated CDAs. In addition, Strieker et al. overproduced AsnO and have shown that the enzyme is a nonhaem $\mathrm{Fe}(\mathrm{II}) / 2$-oxoglutarate-dependent oxygenase that $\beta$-hydroxylates free Asn [10], as we had earlier proposed [9]. Currently, it is suggested that hydroxylation of Asn occurs prior to activation and incorporation into the CDA lipopeptide core and that phosphorylation is most likely to occur during or after lipopeptide assembly (Fig. 4A) [9].

An unusual Z-dehydrotryptophan residue $(Z-\Delta \operatorname{Tr} p)$ is found at the $\mathrm{C}$-terminus of the a-series CDA variants, which is evident from a strong characteristic adsorption at $350 \mathrm{~nm}$ in their UV spectrum [4]. Despite considerable effort, we were unable to identify a gene encoding candidate Trp-oxidase or -dehydrogenase enzymes that may catalyze the oxidation of the C-terminal Trp residue in CDA. In the absence of such a gene or enzyme, we resorted to classical labeling experiments to determine the stereochemical course of Trp dehydrogenation during CDA biosynthesis [11]. In these experiments, synthetic 

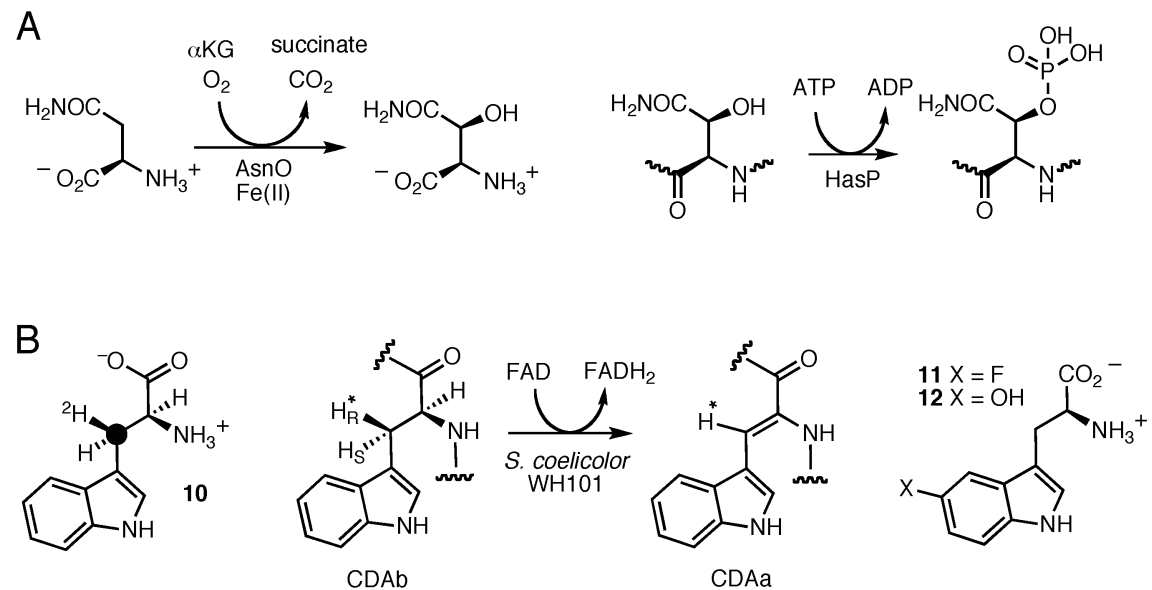

Fig. 4 (A) Biosynthesis of the 3-hydroxyasparagine (3-OHAsn) and 3-phosphohydroxyasparagine (3-OPAsn) residues found at position 9 in CDAs. (B) Stereochemical course of Trp-dehydrogenation during CDA biosynthesis and modified tryptophans $(\mathbf{1 1 , 1 2})$ that were used to develop auxotrophic precursor-directed biosynthesis of modified CDAs.

stereospecifically deuterated Trp precursors, including doubly labeled $\left(2^{\prime} S, 3^{\prime} R\right)-\left[3-{ }^{2} \mathrm{H}_{1},{ }^{13} \mathrm{C}_{1}\right]$-tryptophan 10, were fed to the Trp-auxotrophic strain of S. coelicolor WH101. The use of the auxotroph enabled the fate of the ${ }^{2} \mathrm{H}$ labels during CDA biosynthesis to be determined by MS and NMR. From this it was shown that the hydrogen is abstracted from the $\mathrm{C}^{\prime}$ and $\mathrm{C} 3$ ' pro-S positions of an $(S)$-tryptophanyl precursor, with overall syn stereochemistry. This suggests a direct dehydrogenation catalyzed by a flavin adenine dinucleotide (FAD)-dependent dehydrogenase/oxidase (Fig. 4B) [11]. This information will aid efforts to isolate and characterize the putative Trp-dehydrogenase/oxidase. In addition, using auxotrophic precursor-directed biosynthesis, CDAs possessing modified 5-fluoro- and 5-hydroxy-Trp residues were produced [12] (Fig. 7: HWCDAs and FWCDAs). In these experiments, the S. coelicolor Trp-auxotroph WH101 is grown on minimal media supplemented with synthetic Trp analogs $\mathbf{1 1}$ and 12, which were subsequently incorporated into the CDA lipopeptide. This was one of the first reports describing the application of amino acid auxotrophy in the precursor-directed biosynthesis of nonribosomal peptides with synthetic (non-natural) amino-acid residues. In addition, 5-hydroxytryptophan (5HW) 12 exhibits fluorescence excitation at wavelengths longer than the absorbance of Trp. As a result, 5HW-containing lipopeptides could be selectively excited in vivo, which might be used to study the interactions of lipopeptides with specific molecular targets within bacterial cell membranes.

\section{Trans-2,3-epoxyhexanoyl fatty acid side chain}

There are five genes at the end of the $c d a$ biosynthetic gene cluster (Fig. 2B), which are implicated in the biosynthesis of the trans-2,3-epoxyhexanoyl fatty acid moiety of CDAs [4]. The gene acp encodes an acyl carrier protein (ACP) and $f a b F 3$ and $f a b H 4$ encode proteins that are similar to $\alpha$-ketoacyl-ACP synthase KAS-II and -III enzymes, respectively. Typically, KAS-III enzymes catalyze the first Claisentype condensation reactions in fatty acid biosynthesis, whilst KAS-II catalyzes the second and subsequent condensations. Presumably, these enzymes combine with other fatty acid synthase (FAS) enzymes from primary metabolism to deliver the CDA hexanoyl precursor (Fig. 5). hxcO and hcmO encode proteins that are similar to FAD-dependent oxidase and monooxygenase enzymes, respectively, which are predicted to catalyze the desaturation and epoxidation of the CDA hexanoyl fatty acid precursor $[4,13]$. To explore this, we deleted the $h x c O$ and $h c m O$ genes from the $S$. coelicolor chromosome. In the case of the $\Delta h x c O$ mutant, we were able to isolate several new CDAs, which were shown by tan- 

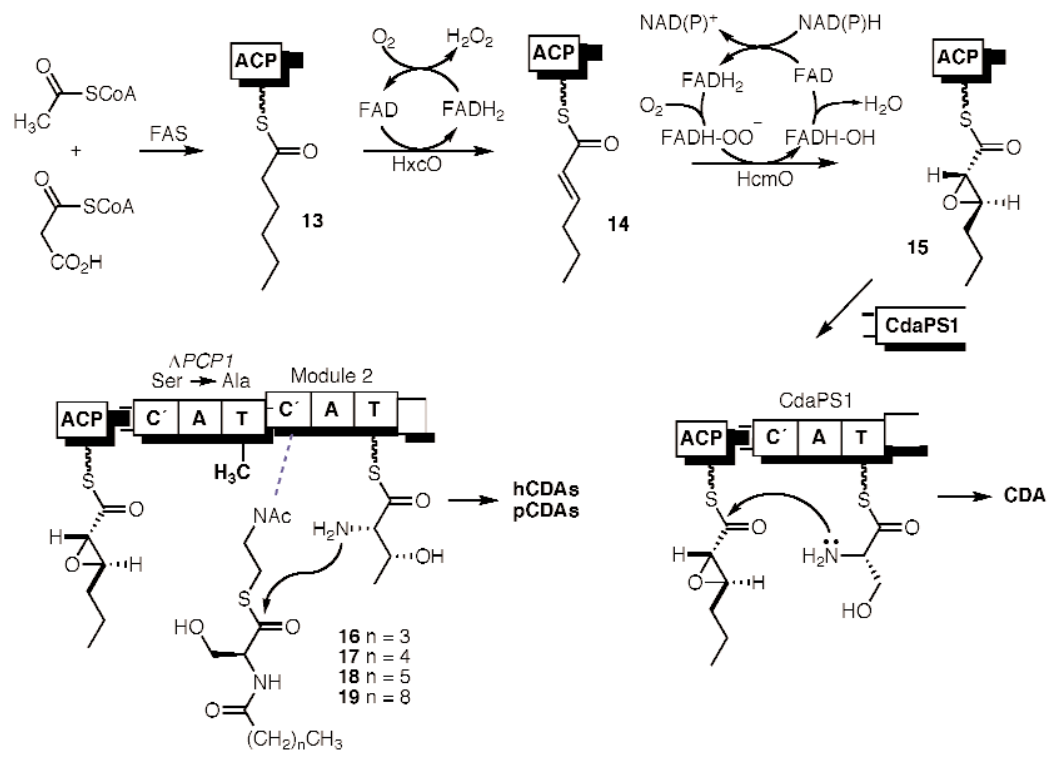

Fig. 5 Biosynthesis of the CDA 2,3-epoxyhexanoyl fatty side chain and transfer of the fatty acid moiety to the CDA NRPS, which is the proposed initiation step in lipopeptide assembly. Also shown is the $\triangle P C P 1$ mutant NRPS, which accepts synthetic $N$-acyl-L-serinyl $N$-acetylcysteamine (NAC) analogs 16 and 17 and leading to CDAs with pentanoyl and hexanoyl side chains (pCDAs and hCDAs).

dem MS experiments to be lipopeptides with hexanoyl fatty acid side chains (Fig. 7, hCDA3b, 4a, and 4b) [13]. In addition, we overproduced $\mathrm{HxcO}$ and $\mathrm{HcmO}$ and showed that these enzymes were unable to process a putative hexanoyl-CoA precursor. This, combined with other evidence, led us to propose that a dedicated FAS delivers an hexanoyl-ACP precursor 13, which is desaturated and epoxidized by the flavin-dependent oxidase $(\mathrm{HxcO})$ and monoxygenase $(\mathrm{HcmO})$ enzymes, respectively. Following this, we suggested that the 2,3-epoxyhexanoyl moiety $\mathbf{1 5}$ is transferred from the ACP to the NRPS, by the C'-domain of module 1 (Fig. 5). Subsequently, Kopp et al. [14] were able to confirm that. HxcO and $\mathrm{HcmO}$ do catalyze desaturation and epoxidation of the hexanoyl-ACP precursor, which was in agreement with our earlier study [13].

Following this, we developed a mutasynthesis approach, for modifying the CDA lipid moiety. This relied on changing the active site Ser to Ala on the peptidyl carrier protein (PCP) domain of the first module of the CDA NRPS. This blocks phosphopantetheinylation of the PCP domain and the subsequent accumulation of intermediates on module 1 of the mutant NRPS $(\triangle P C P 1)$. In the absence of the natural intermediate it was possible to affect incorporation of different exogenous $N$-acyl-L-serinyl $N$-acetylcysteamine (NAC) analogs $(\mathbf{1 6 , 1 7})$, in the $\triangle P C P 1$ strain leading to new lipopeptides with different fatty acid side chains [13]. In addition to generating hexanoyl-CDAs, we showed that feeding $N$ pentanoyl-L-serinyl $\mathrm{N}$-acetylcysteamine (16) gave rise to a new CDA with a pentanoyl side chain (pCDA4b). As far as we are aware, this was the first rational approach to be developed for engineering the fatty acid component of lipopeptides.

Despite this, attempts to incorporate longer acyl chains (>C6), using this approach (e.g., feeding 18 and 19), were unsuccessful, suggesting that the CDA lipopeptide assembly line has evolved tight specificity for C6 or shorter acyl chains. Indeed, CDAs are unusual in possessing such a short highly conserved lipid moiety, compared with the other lipopeptides which possess longer and more variable fatty acid side chains. Through sequence alignment we have identified the enzyme KAS-II, encoded by $f a b F 3$, as a possible fatty acid chain length determinant. On the basis of known structures of related KAS-II enzymes, it is possible to identify the putative acyl binding pockets at the active site. In the case 
of KAS-II from CDA biosynthesis, we suggested that the acyl binding pocket is blocked by the side chain of Phe108, giving rise to the short hexanoyl-ACP precursor. To explore this, we are subjecting KAS-II to site-directed mutagenesis in order to change the specificity of the enzyme for longer fatty acid precursors.

\section{NRPS: Active site modification of A-domains and module/domain exchange}

The precursor-directed biosynthesis and mutasynthesis experiments described above led to new lipopeptide antibiotics incorporating synthetic amino acid residues $[4,8,12,13]$. However, the yields of these new engineered products were often low, which is likely to be due to the tight specificity of the adenylation (A) domain on the NRPS. To address this, we have been investigating modification of the active site of A-domains to enable activation of a wider range of amino acid substrates. Using the $\mathrm{X}$ ray crystal structure of (PheA), a Phe-activating A-domain, from gramicidin S synthetase [15], the key active site residues of each A-domain on the CDA NRPS were identified. Noticeably, the active sites of the Asp-activating A-domains of modules 4, 5, and 7 are all identical to each other and also similar to the module 9 Asn activating A-domain differing at positions 299, 322, and 331 (Fig. 6). Accordingly, a DNA fragment encompassing module 7, was subjected to site directed mutagenesis to generate single (Ala322 $\rightarrow$ Glu) and double-point (Ala322 $\rightarrow$ Glu, Asn331 $\rightarrow$ Gly) mutants that were cloned into plasmids, which were used to deliver the mutations on to the $S$. coelicolor chromosome through homologous recombination [16]. The single-point mutant failed to produce any CDAs with Asn or Asp at position 7. However, a new product was identified by LC-MS which was isolated and subsequently shown to the CDA-hexapeptide intermediate 20 (Fig. 6). On the other hand, the double-point mutant produced both the hexapeptide 20 and a CDA product CDA2a-7N (Fig. 7) possessing Asn at position 7 rather than Asp, that is not seen in the wild type [16].

A

\begin{tabular}{llllllllllll} 
PheA & 235 & 236 & 239 & 278 & 299 & 301 & 322 & 330 & 331 & 517 \\
\hline Asp (4,5 \& 7) & D & L & T & K & I & G & $\underline{A}$ & $V$ & $\underline{N}$ & $K$ \\
Asn (9) & D & L & $\mathrm{T}$ & $\mathrm{K}$ & $\mathrm{V}$ & $\mathrm{G}$ & $\underline{E}$ & $\mathrm{~V}$ & $\underline{\mathrm{G}}$ & $\mathrm{K}$
\end{tabular}

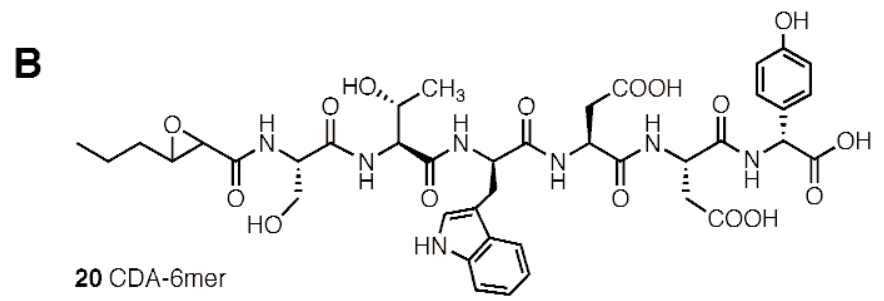

Fig. 6 (A) Sequence alignment of the active site residues of the Asp and Asn activating adenylation domains of the CDA NRPS. Numbered according to the Phe-activating A-domain, from gramicidin S synthetase. (B) The CDA hexapeptide intermediate $\mathbf{2 0}$. 


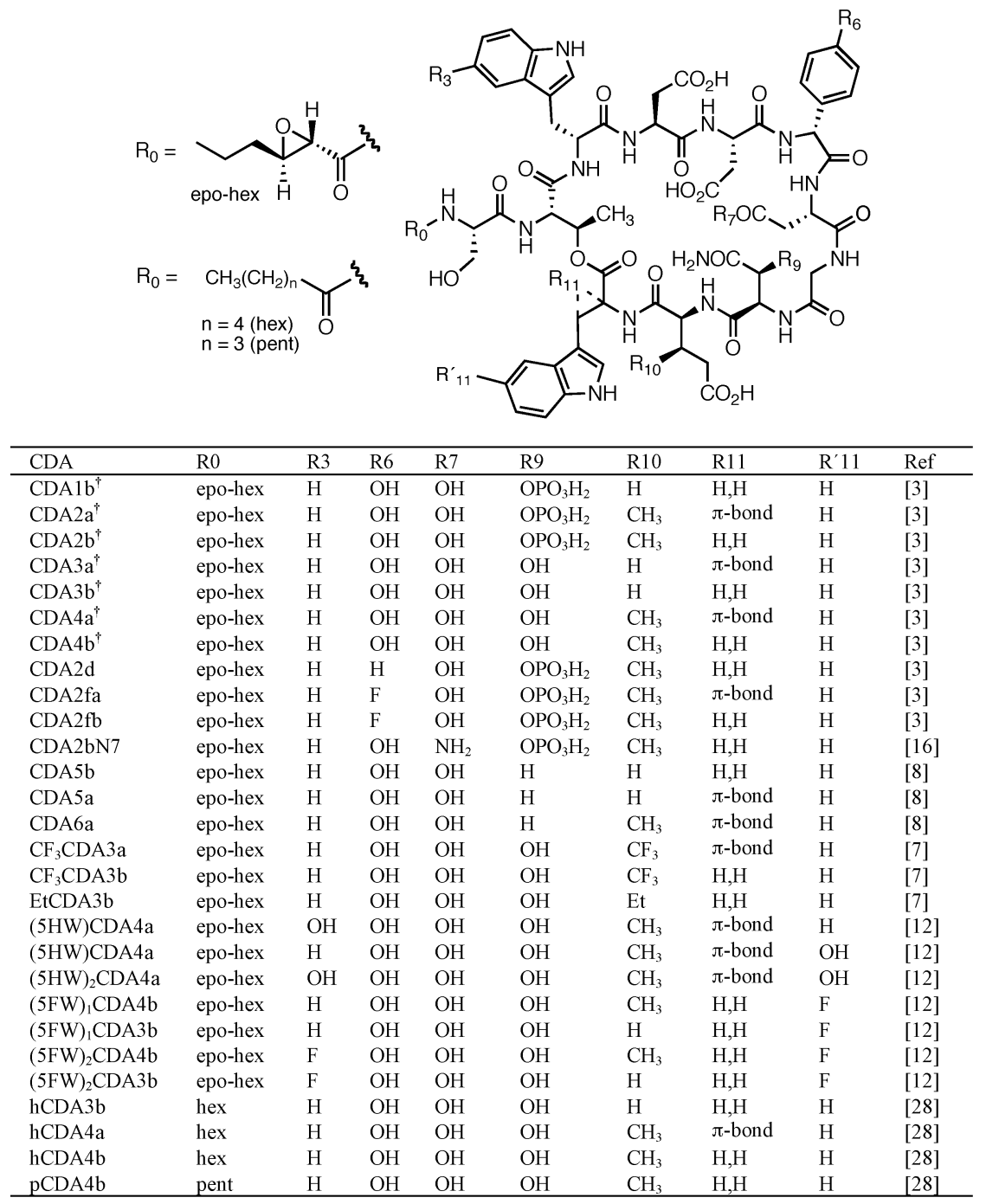

Fig. 7 CDA structural variants that have resulted from the biosynthetic engineering described in this study ${ }^{\dagger}$. Wildtype CDAs.

This was significant as it was one of the first examples of how active site modification of an adenylation domain could be used to change the sequence of a nonribosomal peptide natural product. Interestingly, the extracts containing CDAa-7N are inactive in bioassays, which suggests that Asp-7 is essential for calcium binding and antibiotic activity of CDAs. Finally, the isolation of the CDA hexapeptide intermediate $\mathbf{2 0}$ suggests the possible existence of an NRPS proofreading mechanism. For example, changes to the active site of the A-domain may result in less efficient activation of a non-cognate amino acid substrate, which could cause a kinetic blockage on the NRPS and hydrolysis of the stalled upstream peptidyl thioester. It has been suggested that type II thioesterase enzymes (TeII) may catalyze such hydrolysis reactions. We therefore deleted the corresponding teII gene from the $S$. coelicolor module 7, A-domain single-point mutant. However, this double mutant was also shown to produce the CDA-hexapeptide 20, which suggests that TeII is not responsible for the hydrolysis of the stalled peptidyl intermediate. Instead, we have proposed that an alternative NRPS proofreading mech- 
anism exists where the upstream condensation (C) domain catalyzes the hydrolysis of the stalled peptidyl intermediates. According to this hypothesis, the acceptor-site of the C-domain is less able to recognize the non-cognate substrate. Consequently, a water molecule can bind to the acceptor site and then using the catalytic functionality of the $\mathrm{C}$ domain, intercept the peptidyl-thioester intermediate in the donor site of the C-domain. Industrial scientists at Cubist Pharmaceuticals have also shown that modules and domains in the daptomycin NRPS were exchanged with the corresponding modules from CDAs and other lipoptides, resulting in new daptomycin analogs [17].

\section{CONCLUSION}

Many of the key genes involved in CDA biosynthesis have been identified and deleted from the biosynthetic gene cluster. Through characterization of the lipopepetides produced by these resulting mutants, and by complementation with synthetic intermediates, we have been able to elucidate most of the steps involved in the biosynthesis of CDAs. In addition, a number of the biosynthetic enzymes have been overproduced and shown to demonstrate in vitro activity consistent with their proposed role in CDA biosynthesis in vivo. Finally, a range of different methods have been developed for engineering the combinatorial biosynthesis of CDA-type lipopeptides. For example, mutasynthesis and auxotrophic precursor-directed biosynthesis have been used to modify the fatty acid moiety, as well as HPG-, 3-MeGlu-, and Trp-derived residues in CDAs (Fig. 7). Deletions and modifications of enzymes involved in fatty acid biosynthesis have also resulted in CDAs with altered lipid moieties. Finally, active site modifications of adenylation domains of the NRPS have also been used to change the amino acid sequence of CDAs.

\section{ACKNOWLEDGMENTS}

This work was supported by the BBSRC through research grants 36/B12126 and BB/C50366 2. Biotica is also acknowledged for additional support. The many coworkers and collaborators who contributed to this work are gratefully acknowledged, and their names are included in the original publications listed below.

\section{REFERENCES}

1. B. Wilkinson, J. Micklefield. Nat. Chem. Biol. 3, 379 (2007).

2. L.-J. Ball, C. M. Goult, J. A. Donarski, J. Micklefield, V. Ramesh. Org. Biomol. Chem. 2, 1872 (2004).

3. (a) J. N. Steenbergen, J. Alder, G. M. Thorne, F. P. Tally. J. Antimicrob. Chemother. 55, 283 (2005); (b) S. K. Straus, R. E. W. Hancock. Biochem. Biophys. Acta 1758, 1215 (2006); (c) J. Micklefield. Chem. Biol. 11, 887 (2004).

4. Z. Hojati, C. Milne, B. Harvey, L. Gordon, M. Borg, F. Flett, B. Wilkinson, C. P. Smith, J. Micklefield. Chem. Biol. 9, 1175 (2002).

5. (a) O. W. Choroba, D. H. Williams, J. B. Spencer. J. Am. Chem. Soc. 122, 5389 (2000); (b) B. K. Hubbard, M. G. Thomas, C. T. Walsh. Chem. Biol. 7, 931 (2000).

6. C. Milne, A. Powell, J. Jim, M. Al Nakeeb, C. P. Smith, J. Micklefield. J. Am. Chem. Soc. 128, 11250 (2006).

7. C. Mahlert, F. Kopp, J. Thirlway, J. Micklefield, M. A. Marahiel. J. Am. Chem. Soc. 129, 12011 (2007).

8. A. Powell, M. Al Nakeeb, B. Wilkinson, J. Micklefield. Chem. Commun. 2683 (2007).

9. J. Neary, A. Powell, L. Gordon, C. Milne, F. Flett, B. Wilkinson, C. P. Smith, J. Micklefield. Microbiology 153, 768 (2007).

10. M. Strieker, F. Kopp, C. Mahlert, L.-O. Essen, M. A. Marahiel. Chem. Biol. 187 (2007).

(C) 2009 IUPAC, Pure and Applied Chemistry 81, 1065-1074 
11. (a) B. A. Heidari, J. Thirlway, J. Micklefield. Org. Lett. 9, 1513 (2007); (b) B. A. Heidari, J. Micklefield. J. Org. Chem. 72, 8950 (2007).

12. B. A. Heidari, J. Thirlway, J. Micklefield. Org. Biomol. Chem. 6, 975 (2008).

13. A. Powell, M. Borg, B. Amir -Heidari, J. M. Neary, J. Thirlway, B. Wilkinson, C. P. Smith, J. Micklefield. J. Am. Chem. Soc. 129, 15182 (2007).

14. F. Kopp, U. Linne, M. Oberthür, M. A. Marahiel. J. Am. Chem. Soc. 130, 2656 (2008).

15. (a) T. Stachelhaus, H. D. Mootz, M. A. Marahiel. Chem. Biol. 6, 493 (1999); (b) G. L. Challis, J. Ravel, C. A. Townsend. Chem. Biol. 7, 211 (2000).

16. G. C. Uguru, C. Milne, M. Borg, F. Flett, C. P. Smith, J. Micklefield. J. Am. Chem. Soc. 126, 5032 (2004).

17. (a) K. Nguyen, D. Ritz, J.-Q. Gu, D. Alexander, M. Chu, V. Miao, P. Brian, R. H. Baltz. Proc. Natl. Acad. Sci. USA 103, 17462 (2006); (b) V. Miao, M.-F. Coëffet-Le Gal, K. Nguyen, P. Brian, J. Penn, A. Whiting, J. Steele, D. Kau, S. Martin, R. Ford, T. Gibson, M. Bouchard, S. K. Wrigley, R. H. Baltz. Chem. Biol. 13, 269 (2006); (c) M.-F. Coëffet, Le Gal, L. Thurson, P. Rich, V. Miao, R. H. Baltz. Microbiology 152, 2993 (2006). 